\title{
Triterpenoid Saponins from the Roots of Pulsatilla koreana
}

Seong-Cheol Bang, ${ }^{\dagger \neq}$ Yong Kim ${ }^{\dagger}$ Jee-Hyun Lee, ${ }^{\dagger}$ and Byung-Zun Ahn ${ }^{*}{ }^{\dagger}$

Central Research Institute, SB Pharmaceutical Co. (Ltd), Gongju 314-923, Chungnam, Korea and

College of Pharmacy, Chungnam National University, 305-764 Daejon, Korea

Supporting Information:

Isolation Procedure for Known Substances from Pulsatilla koreana Roots

\footnotetext{
* To whom inquiries should be addressed. Tel: (82)-41-857-3686. Fax: (82)41-857-7854. E-mail: ahnbj@cnu.ac.kr.

${ }^{\dagger}$ Central Research Institute, SB Pharmaceutical Co. (Ltd), Korea.

* College of Pharmacy, Chungnam National University, Korea.
} 


\section{Supporting Information}

\section{Isolation Procedure for Known Substances from Pulsatilla koreana Roots}

Fractions $1(3.1 \mathrm{~g})$ and $2(2.4 \mathrm{~g})$ were subjected to silica gel column chromatography $(350 \mathrm{~g}, 80 \times 4.8$ $\mathrm{cm}$ ) eluting with a stepwise gradient mixture of $\mathrm{CHCl}_{3}-\mathrm{MeOH}-\mathrm{H}_{2} \mathrm{O}$ $(100: 0: 0 \rightarrow 90: 10: 0 \rightarrow 80: 20: 0 \rightarrow 60: 30: 5)$, to give two subfractions; fraction 1-1 (0.91 $\mathrm{g}, R_{f}=0.08$ in $\left.\mathrm{CHCl}_{3}-\mathrm{MeOH}-\mathrm{H}_{2} \mathrm{O}=6: 3: 0.5\right)$ and fraction 2-1 (0.78 g, $R_{f}=0.2$ in $\left.\mathrm{CHCl}_{3}-\mathrm{MeOH}-\mathrm{H}_{2} \mathrm{O}=6: 3: 0.5\right)$, respectively. The further separation of fraction 1-1 was performed by HPLC using a Mightysil column (250 mm $\times 10 \mathrm{~mm}, \mathrm{RP}_{-} \mathrm{C}_{18}, 5 \mu \mathrm{m}$, Kanto, Japan) eluting with $80 \% \mathrm{MeOH}$ (flow rate: $1 \mathrm{~mL} / \mathrm{min}$ ), to yield 23-hydroxy-3 $\beta$-[( $O$ - $\alpha$-L-rhamnopyranosyl- $(1 \rightarrow 2)-O$ - $[O$ - $\beta$-D-glucopyranosyl- $(1 \rightarrow 4)]$ - $\alpha$-L-

arabinopyranosyl)oxy]lup-20(29)-en-oic acid 28- $O$ - $\alpha$-L-rhamnopyranosyl-(1 $\rightarrow 4$ )- $O$ - $\beta$-D-glucopyranosyl$(1 \rightarrow 6)$ - $\beta$-D-glucopyranosyl ester $\left(145.0 \mathrm{mg}, R_{t}=13.8 \mathrm{~min}\right)^{6}$ and 3- $O$ - $\alpha$-L-rhamnopyranosyl- $(1 \rightarrow 2)$ - $[\beta$-Dglucopyranosyl-( $1 \rightarrow 4)]$ - $\alpha$-L-arabinopyranosyl hederagenin $\quad 28-O$ - $\alpha$-L-rhamnopyranosyl-( $1 \rightarrow 4)-\beta$-Dglucopyranosyl-( $1 \rightarrow 6)$ - $\beta$-D-glucopyranosyl ester $\left(70.5 \mathrm{mg}, R_{t}=18.6 \mathrm{~min}\right){ }^{7}$ Then, fraction $2-1$ was further separated, under the same conditions as fraction 1-1, to afford 23-hydroxy-3 $\beta$-[( $O-\alpha$-L-rhamnopyranosyl$(1 \rightarrow 2)$ - $O$ - $\alpha$-L-arabinopyranosyl)oxy]lup-20(29)-en-oic acid 28 - $O$ - $\alpha$-L-rhamnopyranosyl-(1 $\rightarrow 4)-O$ - $\beta$-Dglucopyranosyl-( $1 \rightarrow 6)$ - $\beta$-D-glucopyranosyl ester $\left(164.6 \mathrm{mg}, \quad R_{t}=14.2 \mathrm{~min}\right)^{6}$ and $3-O-\alpha$-Lrhamnopyranosyl-( $1 \rightarrow 2)$ - $\alpha$-L-arabinopyranosyl] hederagenin $28-O$ - $\alpha$-L-rhamnopyranosyl-( $1 \rightarrow 4)-\beta$-Dglucopyranosyl-( $1 \rightarrow 6)$ - $\beta$-D-glucopyranosyl ester $\left(35.7 \mathrm{mg}, R_{t}=17.4 \mathrm{~min}\right){ }^{7}$

Fraction 3, $3.7 \mathrm{~g}$ was loaded into a silica gel column $(300 \mathrm{~g}, 80 \times 4.8 \mathrm{~cm})$ and eluted with a mixture of $\mathrm{CHCl}_{3}-\mathrm{MeOH}-\mathrm{EtOAc}-\mathrm{H}_{2} \mathrm{O}$ (2:2:4:1, lower phase), to give fractions 3-1 (0.85 g, $R_{f}=0.30$ in eluting solvent condition), 3-2 (1.23 g, $\left.R_{f}=0.16\right)$, and 3-3 (0.55 g, $\left.R_{f}=0.09\right)$. The further separation of each of the subfractions 3-1, 3-2, and 3-3 was performed by successive HPLC using a preparative Spherisorb ${ }^{\circledR}$ S5 ODS2 column (250 mm $\times 10 \mathrm{~mm}, \mathrm{RP}_{-} \mathrm{C}_{18}, 5 \mu \mathrm{m}$, Waters, Milford, MA), eluting with a mixture of MeCN and $\mathrm{H}_{2} \mathrm{O}$. Fraction 3-1 (0.85 g) was dissolved in $10 \mathrm{~mL} \mathrm{MeOH}$ and filtered on a $0.45 \mu \mathrm{m}$ membrane filter. An aliquot $(100 \mu \mathrm{L})$ of the $\mathrm{MeOH}$ solution was subjected to preparative HPLC eluting with $43 \% \mathrm{MeCN}$ (flow rate: $3 \mathrm{~mL} / \mathrm{min}$ ), to give the pure known compounds hederagenin 3 - $O$ - $\beta$-D-glucopyranosyl-(1 $\rightarrow 3)$ $\alpha$-L-rhamnopyranosyl-( $1 \rightarrow 2)$ - $\alpha$-L-arabinopyranoside $\left(121.1 \mathrm{mg}, R_{t}=18.6 \mathrm{~min}\right){ }^{8}$, oleanolic acid 3-O- $\alpha$-Lrhamnopyranosyl-( $1 \rightarrow 2)$-[ $\beta$-D-glucopyranosyl-(1 $\rightarrow 4)]$ - $\alpha$-L-arabinopyranoside $\left(21.7 \mathrm{mg}, R_{t}=27.1 \mathrm{~min}\right){ }^{8}$ oleanolic acid 3-O- $\beta$-D-glucopyranosyl-( $1 \rightarrow 3)$ - $\alpha$-L-rhamnopyranosyl-( $1 \rightarrow 2)$ - $\alpha$-L-arabinopyranoside (39.6 mg, $\left.R_{t}=36.3 \mathrm{~min}\right),{ }^{8}$ and hederagenin $3-O-\beta$-D-glucopyranosyl-( $\left.1 \rightarrow 4\right)$ - $\alpha$-L-arabinopyranoside $(17.1$ $\left.\mathrm{mg}, R_{t}=46.0 \mathrm{~min}\right) .^{9}$ In addition, hederagenin 3-O- $\alpha$-L-rhamnopyranosyl-(1 $\left.\rightarrow 2\right)$-[ $\beta$-D-glucopyranosyl$(1 \rightarrow 4)$ ]- $\alpha$-L-arabinopyranoside $\left(310 \mathrm{mg}, R_{t}=43.5 \mathrm{~min}\right){ }^{7}$ obtained from fraction $3-2$, was separated using the same preparative HPLC method using 40\% $\mathrm{MeCN}$ (flow rate: $1.5 \mathrm{~mL} / \mathrm{min}$ ).

Fraction 4 was subjected to silica gel column chromatography eluting with a mixture of $\mathrm{CHCl}_{3}-\mathrm{MeOH}-$ 
EtOAc- $\mathrm{H}_{2} \mathrm{O}$ (2:2:4:1, lower phase), to give subfraction 4-1 (0.73 g, $R_{f}=0.08$ in eluting solvent condition). The further separation of fraction 4-1 was performed by HPLC used a preparative Spherisorb ${ }^{\circledR}$ S5 ODS2 column eluting with $45 \% \mathrm{MeCN}$ (flow rate: $2 \mathrm{~mL} / \mathrm{min}$ ), to give 23 -hydroxy-3 $\beta-[(O-\alpha$ - Lrhamnopyranosyl-( $1 \rightarrow 2)$ - $O$ - $\alpha$-L-arabinopyranosyl)oxy]lup-20(29)-en-oic acid $\left(200.8 \mathrm{mg}, R_{t}=27.1 \mathrm{~min}\right)^{6}$ and hederagenin 3- $O$ - $\alpha$-L-rhamnopyranosyl-(1 $\rightarrow 2)$ - $\alpha$-L-arabinopyranoside $\left(160.9 \mathrm{mg}, R_{t}=30.8 \mathrm{~min}\right){ }^{8}$ The identification of these known compounds was supported by $1 \mathrm{D}$ and $2 \mathrm{D}$ NMR $\left({ }^{13} \mathrm{C}\right.$ NMR, ${ }^{1} \mathrm{H}$ NMR, ${ }^{1} \mathrm{H}-{ }^{1} \mathrm{H}$ COSY, HMQC and HMBC) methods, FABMS, and hydrolysis. 